\title{
ACCELERATION AND TRAPPING OF PARTICLES BY RADIATION PRESSURE
}

\author{
A. Ashkin \\ Bell Telephone Laboratories, Holmdel, New Jersey 07733 \\ (Received 3 December 1969)
}

\begin{abstract}
Micron-sized particles have been accelerated and trapped in stable optical potential wells using only the force of radiation pressure from a continuous laser. It is hypothesized that similar accelerations and trapping are possible with atoms and molecules using laser light tuned to specific optical transitions. The implications for isotope separation and other applications of physical interest are discussed.
\end{abstract}

This Letter reports the first observation of acceleration of freely suspended particles by the forces of radiation pressure from $\mathrm{cw}$ visible laser light. The experiments, performed on micron-sized particles in liquids and gas, have yielded new insights into the nature of radiation pressure and have led to the discovery of stable optical potential wells in which particles were trapped by radiation pressure alone. The ideas can be extended to atoms and molecules where one can predict that radiation pressure from tunable lasers will selectively accelerate, trap, or separate the atoms or molecules of gases because of their large effective cross sections at specific resonances. The author's interest in radiation pressure from lasers stems from a realization of the large magnitude of the force, and the observation that it could be utilized in a way which avoids disturbing thermal effects. For instance a power $P=1 \mathrm{~W}$ of $\mathrm{cw}$ argon laser light at $\lambda=0.5145 \mu \mathrm{m}$ focused on a lossless dielectric sphere of radius $r=\lambda$ and density $=1$ $\mathrm{gm} / \mathrm{cc}$ gives a radiation pressure force $F_{\mathrm{rad}}$ $=2 q P / c=6.6 \times 10^{-5} \mathrm{dyn}$, where $q$, the fraction of light effectively reflected back, is assumed to be of order 0.1 . The acceleration $=1.2 \times 10^{8} \mathrm{~cm} /$ $\sec ^{2} \cong 10^{5}$ times the acceleration of gravity.

Historically, ${ }^{1,2}$ the main problem in studying radiation pressure in the laboratory has been the obscuring effects of thermal forces. These are caused by temperature gradients in the medium surrounding an object and, in general, are termed radiometric forces. ${ }^{3}$ When the gradients are caused by light, and the entire particle moves, the effect is called photophoresis. ${ }^{3,4}$ These forces are usually orders of magnitude larger than radiation pressure. Even with lasers, photophoresis usually completely obscures radiation pressure. ${ }^{5}$ In our work, radiometric effects were avoided by suspending relatively transparent particles in relatively transparent media. We operated free of thermal effects at $10^{3}$ times the power densities of Ref. 5 .

The first experiment used transparent latex spheres ${ }^{6}$ of $0.59-, 1.31-$, and $2.68-\mu \mathrm{m}$ diam freely suspended in water. $\mathrm{A} \mathrm{TEM}_{00}$-mode beam of an argon laser of radius $w_{0}=6.2 \mu \mathrm{m}$ and $\lambda$ $=0.5145 \mu \mathrm{m}$ was focused horizontally through a glass cell $120 \mu \mathrm{m}$ thick and manipulated to focus on single particles. See Fig. 1(a)。 Results were observed with a microscope. If a beam with milliwatts of power hits a 2.68- $\mu \mathrm{m}$ sphere off center, the sphere is simultaneously drawn in to the beam axis and accelerated in the direction of the light. It moves with a limiting velocity of microns per second until it hits the front surface of the glass cell where it remains trapped in the beam. If the beam is blocked, the sphere wanders off by Brownian motion. Similar effects occur with the other sphere sizes but more power is required for comparable velocities. When mixed, one can accelerate $2.68-\mu \mathrm{m}$ spheres and leave $0.585-\mu \mathrm{m}$ spheres behind. The particle velocities and the trapping on the beam axis can

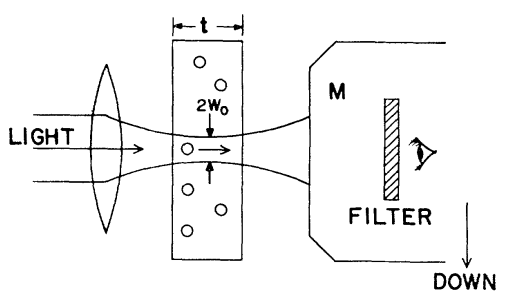

(a)

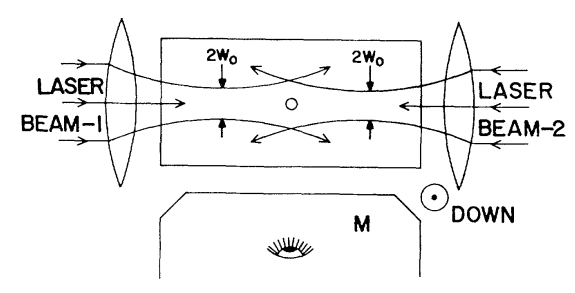

(b)

FIG. 1. (a) Geometry of glass cell, $t=120 \mu \mathrm{m}$, for observing micron particle motions in a focused laser beam with a microscope $M$. (b) The trapping of a highindex particle in a stable optical well. Note position of the $\mathrm{TEM}_{00}$-mode beam waists. 
be understood as follows (see Fig. 2): The sphere of high index $n_{H}=1.58$ is situated off the beam axis, in water of lower index $n_{L}=1.33$. Consider a typical pair of rays symmetrically situated about the sphere axis $B$. The stronger ray (a) undergoes Fresnel reflection and refraction (called a deflection here) at the input and output faces. These result in radiation pressure forces $F_{R}{ }^{i}, F_{R}{ }^{\circ}$ (the input and output reflection forces), and $F_{D}{ }^{i}, F_{D}{ }^{\circ}$ (the input and output deflection forces), directed as shown. Although the magnitudes of the forces vary considerably with angle $\Phi$, qualitatively the results are alike for all $\Phi$. The radial $(r)$ components of $F_{D}{ }^{i}, F_{D}{ }^{\circ}$ are much larger than $F_{R}{ }^{i}$ and $F_{R}{ }^{\circ}$ (by $\sim 10$ at $\Phi$ $\left.=25^{\circ}\right)$. All forces give accelerations in the $+z$ direction. $F_{R}{ }^{i}$ and $F_{R}{ }^{\circ}$ cancel radially to first order. $F_{D}{ }^{i}$ and $F_{D}{ }^{\circ}$ add radially in the $-r$ direction, thus the net radial force for the stronger ray is inward toward higher light intensity. Similarly the symmetrical weak ray $(b)$ gives a net force along $+z$ and a net outward but weaker radial force. Thus the sphere as a whole is accelerated inward and forward as observed. To compute the $z$ component of the force for a sphere on axis, one integrates the perpendicular $(s)$ and parallel $(p)$ components of the plane-polarized beam over the sphere. This yields an effective $q=0.062$ 。 This geometric optic result (neglecting diffraction) is identical with the asymptotic limit of a wave analysis by Debye $e^{2}$ for an incident plane wave. He finds $q=0.06$. From the force we get the limiting velocity $v$ in a viscous medium using Stokes's law. For $r \ll w_{0}$,

$$
v=2 \boldsymbol{q} \operatorname{Pr} / 3 c \pi w_{0}{ }^{2} \eta,
$$

where $\eta$ is the viscosity。 For $P=19 \mathrm{~mW}, w_{0}$ $=6.2 \mu \mathrm{m}$, and a sphere of $r=1.34 \mu \mathrm{m}$ in water $\left(\eta=1 \times 10^{-2} \mathrm{P}\right)$, one computes $v=29 \mu \mathrm{m} / \mathrm{sec}$. We measured $v=26 \pm 5 \mu \mathrm{m} / \mathrm{sec}$ which is good agreement. In the above, the sphere acts as a focusing lens. If one reverses the relative magnitudes of the indices of the media, the sphere becomes a diverging lens, the sign of the radial deflection forces reverse, and the sphere should be pushed out of the beam. This prediction was checked experimentally in an extreme case of a low-index sphere in a high-index medium, namely an air bubble. Bubbles, of $\sim 8-\mu \mathrm{m}$ diam, were generated by shaking a high-viscosity medium consisting of an $80 \%$ by weight mixture of glycerol in water. It was found that the bubbles were always pushed out of the light beam as they were accelerated along, as expected. In the same

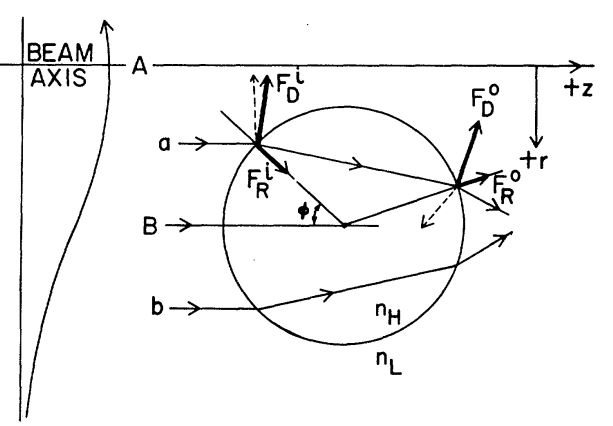

FIG. 2. A dielectric sphere situated off the axis $A$ of a $\mathrm{TEM}_{00}$-mode beam and a pair of symmetric rays $a$ and $b$. The forces due to $a$ are shown for $n_{H}>n_{L}$. The sphere moves toward $+z$ and $-r$.

medium of $n=1.44$, the 2.68- $\mu \mathrm{m}$ spheres of $n$ $=1.58$ were still focusing. At higher powers the bubbles are expected to deform. This would result in a deformation contribution to the radiation pressure force as postulated by Askaryon. ${ }^{7}$ Our observation of the attraction of high-index spheres into regions of high light intensity is related to the deformation of a liquid surface postulated by Kats and Kantorovich. ${ }^{8}$

The experimentally observed radial inward force on the high-index spheres suggest a means of constructing a true optical potential well or "optical bottle" based on radiation pressure alone. If one has two opposing equal $\mathrm{TEM}_{00}$ Gaussian beams with beam waists located as shown in Fig. 1(b), then a sphere of high index will be in stable equilibrium at the symmetry point as shown (i.e., any displacement gives a restoring force). Such trapping was observed experimentally in an open cell filled with $2.68-\mu \mathrm{m}$ spheres in water as sketched in Fig. 1(b). Here the entire beam is viewed at once. Particles are observed by their brilliant scattered light. With $128 \mathrm{~mW}$ in only one of the beams, a maximum particle velocity of $\sim 220 \mu \mathrm{m} / \mathrm{sec}$ was observed as particles traversed the entire near field. The calculated velocity is $195 \mu \mathrm{m} / \mathrm{sec}$. For trapping, the two opposing beams were introduced. Particles that drift near either beam are drawn in, accelerated to the stable equilibrium point, and stop. To check for stability one can interrupt one beam for a moment. This causes the particle to accelerate rapidly in the remaining beam. When the opposing beam is turned on again the particle returns to its equilibrium point, only more slowly since it is now acted on by the differential force. Interrupting the other beam reverses the behavior. In other experiments, $\sim 5-$ $\mu \mathrm{m}$-diam water droplets from an atomizer were 
accelerated in air with a single beam. At 50 $\mathrm{mW}$, velocities $\sim 0.25 \mathrm{~cm} / \mathrm{sec}$ were observed. Such motions could be seen with the naked eye. The behavior of the droplets was in qualitative agreement with expectation.

In our experiments it is clear that we have discriminated against radiometric forces. These forces push more strongly on hot surfaces and would push high-index spheres and bubbles out of the beam; whereas our high-index spheres were drawn into the beam. Even the observed direction of acceleration along the beam axis is the opposite of the radiometric prediction. A moderately absorbing focusing sphere concentrates more heat on the downstream side of both the ball and the medium and should move upstream into the light (negative photophoresis). ${ }^{9}$ For water drops in air we can invoke the wellconfirmed formula of Hettne $\mathrm{r}^{10}$ and compute the temperature gradient needed across a $5-\mu \mathrm{m}$ droplet to account radiometrically for the observed velocity of $0.25 \mathrm{~cm} / \mathrm{sec}$. From Stokes's formula, $F=2.1 \times 10^{-7}$ dyn. Hettner's formula then requires a gradient of $0.5^{\circ} \mathrm{C}$ across the droplet. No such gradients are possible with the $50 \mathrm{~mW}$ used. For water and glycerol the gradients are also very low.

The extension to vacuum of the present experiments on particle trapping in potential wells would be of interest since then any motions are frictionless. Uniform angular acceleration of trapped particles based on optical absorption of circular polarized light or use of birefringent particles is possible. Only destruction by mechanical failure should limit the rotational speed. In vacuum, particles will heat until they are cooled by thermal radiation or vaporize. With the minimum power needed for levitation, micron spheres will assume temperatures of hundreds to thousands of degrees depending on the loss. The ability to heat in vacuum without contaminating containing vessels is of interest. Acceleration of neutral spheres to velocities $\sim 10^{6}$ $10^{7} \mathrm{~cm} / \mathrm{sec}$ is readily possible using powers that avoid vaporization. In this regard one could attempt to observe and use the resonances in radiation pressure predicted by Debye ${ }^{2}$ for spheres with specific radii. The separation of micronor submicron-sized particles by radiation pressure based on radius as demonstrated experimentally could also be useful [see Eq. (1)].

Finally, the extension of the ideas of radiation pressure from laser beams to atoms and molecules opens new possibilities. In general, atoms and molecules are quite transparent. However, if one uses light tuned to a particular transition, the interaction cross section can be much larger than geometric. For example, an atom of sodium has $\pi r^{2}=1.1 \times 10^{-15} \mathrm{~cm}^{2}$ whereas, from the absorption coefficient, ${ }^{11}$ the cross section $\sigma_{T}$ at temperature $T$ for the $D_{2}$ resonance line at $\lambda$ $=0.5890 \mu \mathrm{m}$ is $\sigma_{T}=1.6 \times 10^{-9} \mathrm{~cm}^{2}=0.5 \lambda^{2}$ for $T$ $<40^{\circ} \mathrm{K}$ (region of negligible Doppler broadening). The absorption and isotropic reradiation by spontaneous emission of resonance radiation striking an atom results in an average driving force or pressure in the direction of the incident light. We shall attempt to show that radiation pressure from a laser beam on resonance can work as an actual optical gas pump and operate against significant gas pressures. Figure 3(a) shows a schematic version of such a pump. Imagine two chambers initially filled with sodium vapor, for example. A transparent pump tube of radius $w_{0}$ is uniformly filled with laser light tuned to the $\mathrm{D}_{2}$ line of $\mathrm{Na}$ from the left. Let the total optical power $P$ and the pressure $p_{0}$ be low enough to neglect light depletion and absorption saturation. Most atoms are in the ground state. The average force on an atom is $P \sigma_{T} / c \pi w_{0}^{2}$ and is constant along the pump. Call $x_{\mathrm{cr}}$ the critical distance. It is the distance traveled by an atom in losing its average kinetic energy $\frac{1}{2} m v_{\mathrm{av}}{ }^{2}$. That is, $F x_{\mathrm{cr}}$ $=\frac{1}{2} m v_{\mathrm{av}}{ }^{2} \cong k T$. The variation of pressure in a gas
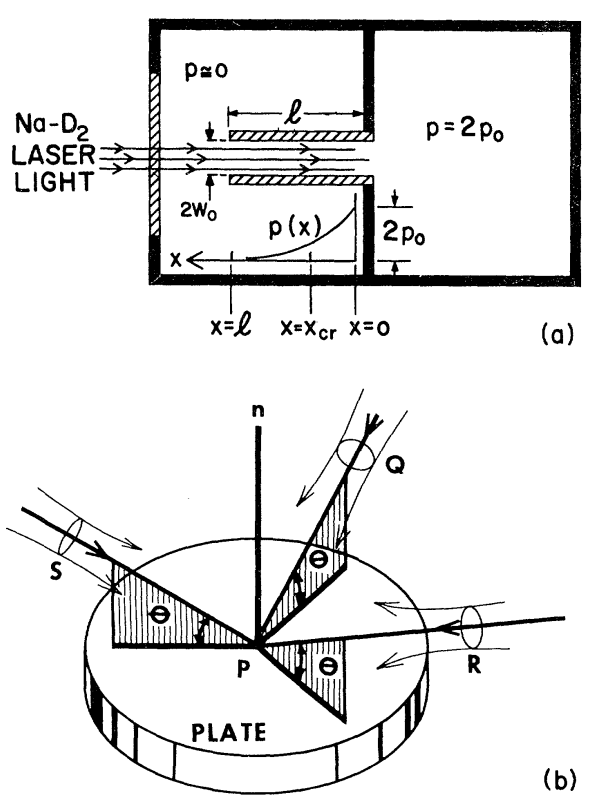

FIG. 3. (a) Schematic optical gas pump and graph of Na pressure $p(x)$. (b) Geometry of gas confinement about point $P$ of a plane surface. 
with a constant force is exponential at equilibrium. Thus

$$
\begin{aligned}
& p(x)=p_{0} e^{-F x / k T}=p_{0} e^{-x / x_{\mathrm{cr}}}, \\
& x_{\mathrm{cr}}=\pi w_{0}{ }^{2} c k T / P \sigma_{T} .
\end{aligned}
$$

Next, consider higher power. Saturation sets in. Population equalization occurs between upper and lower levels for those atoms of the Doppler-broadened line of width $\Delta \nu_{\mathrm{D}}$, within the natural width $\Delta \nu_{\mathrm{n}}$ of line center. A "hole" is burned in the absorption line and the power penetrates more deeply into the gas. But there is a net absorption, even when saturated, due to the everpresent spontaneous emission from the upper energy level. The average force per atom also saturates and is constant along the tube. Its value is $\left(h / \tau_{n} \lambda\right)\left(\Delta \nu_{n} / \Delta \nu_{D}\right)$, where $\tau_{n}$ is the upper level natural lifetime. ${ }^{12}$ Lastly, we consider the effect of collision broadening due to a buffer gas on the force per atom. With collision one replaces $1 / \tau_{\mathrm{n}}$ by $\left(1 / \tau_{\mathrm{n}}+1 / \tau_{\mathrm{L}}\right)$ and $\Delta \nu_{\mathrm{n}}$ by $\left(\Delta \nu_{\mathrm{n}}\right.$ $\left.+\Delta \nu_{\mathrm{L}}\right)$ in the average saturated force, where $\Delta \nu_{\mathrm{L}}$ $=\frac{1}{2} \pi \tau_{\mathrm{L}}$ is the Lorentz width. This enhances the force greatly. Then

$$
x_{\mathrm{cr}}=\frac{k T \lambda}{h}\left(\frac{\tau_{\mathrm{n}} \tau_{\mathrm{L}}}{\tau_{\mathrm{n}}+\tau_{\mathrm{L}}}\right)\left(\frac{\Delta \nu_{\mathrm{D}}}{\Delta \nu_{\mathrm{n}}+\Delta \nu_{\mathrm{L}}}\right) .
$$

As an example, consider Na vapor at $p_{0}=10^{-3}$ Torr $\left(n_{0}=3.4 \times 10^{13}\right.$ atoms $/ \mathrm{cc}$ and $\left.T=510^{\circ} \mathrm{K}\right)$, buffered by helium at 30 Torr. Take a tube of $l=20$ $\mathrm{cm}$ with diameter $2 w_{0}=10^{-2} \mathrm{~cm}$. For $\tau_{n}=1.48$ $\times 10^{-8} \mathrm{sec}, \Delta \nu_{\mathrm{D}}=155 \Delta \nu_{\mathrm{n}}\left(\right.$ at $\left.T=510^{\circ} \mathrm{K}\right)$, and $\Delta \nu_{\mathrm{L}}$ $\cong 30 \Delta \nu_{\mathrm{n}},{ }^{13}$ one finds $x_{\mathrm{cr}}=1.5 \mathrm{~cm}$ and $l=20 \mathrm{~cm}$ $=13.3 x_{\mathrm{cr}}$. Thus $p(l)=2 p_{0} e^{-13.3}=2 \times 10^{-3} \times 1.7$ $\times 10^{-6}=3.4 \times 10^{-9}$ Torr. Essentially complete separation has occurred. This requires a total number of photons per second of $2 \pi \omega_{0}^{2} x_{\mathrm{cr}} n_{0} /\left(1 / \tau_{\mathrm{n}}\right.$ $\left.+1 / \tau_{\mathrm{L}}\right) \cong 1.7 \times 10^{19} \cong 6 \mathrm{~W}$. Under saturated conditions there is little radiation trapping of the scattered light. Almost all the incident energy leaves the gas without generating heat. The technique applies for any combination of gases. Even different isotopes of the same atom or molecule could be separated by virtue of the isotope shift of the resonance lines. The possibilities for forming atomic or molecular beams with specific energy states and for studying chemical reaction kinetics are clear. The possibility of obtaining significant population inversions by resonant gas pumping remains to be evaluated. One can also show that gas can be optically trapped at the surface of a transparent plate. For example [see Fig. 3(b)], three equal $\mathrm{TEM}_{00}$-mode beams with waists at points $Q, R$, and $S$ directed equilaterally at point $P$, at some angle $\theta$, result in a restoring force for displacements of an atom about $P$. Gas trapped about $P$ could serve as a windowless gas target in many experimental situations. The perfection of accurately controlled frequency-tunable lasers is crucial for this work.

It is a pleasure to acknowledge stimulating conversations with many colleagues; in particular, J. G. Bergman, E. P. Ippen, J. E. Bjorkholm, J. P. Gordon, R. Kompfner, and P. A. Wolff. I thank J. M. Dziedzic for making his equipment and skill available.

\footnotetext{
${ }^{1}$ E. F. Nichols and G. F. Hull, Phys. Rev. 17, 26, 91 (1903).

${ }^{2}$ P. Debye, Ann. Physik 30, 57 (1909).

${ }^{3}$ N. A. Fuchs, The Mechanics of Aerosols (The Macmillan Company, New York, 1964).

${ }^{4}$ F. Ehrenhaft and E. Reeger, Compt. Rend 232, 1922 (1951).

${ }^{5}$ A. D. May, E. G. Rawson, and E. H. Hara, J Appl. Phys. 38, 5290 (1967); E. G. Rawson and E. H. May, Appl. Phys. Letters 8, 93 (1966).

${ }^{6}$ Available from the Dow Chemical Company.

${ }^{7}$ G. A. Askar'yan, Zh. Eksperim. i Teor. Fiz.-Pis'ma Redakt. $\underline{9}, 404$ (1969) [translation: JETP Letters $\underline{9}$, $241(1969)]$.

${ }^{8}$ A. V. Kats and V. M. Kantorovich, Zh. Eksperim. i Teor. Fiz.-Pis'ma Redakt. 9, 192 (1969) [translation: JETP Letters $\underline{9}, 112$ (1969)].

${ }^{9}$ See Ref. 3, p. 60.

${ }^{10}$ G. Z. Hettner, Physics 37, 179 (1926); Ref. 3, p. 57.

${ }^{11}$ A. C. G. Mitchell and M. W. Zemansky, Resonance Radiation and Excited Atoms (Cambridge University Press, New York, 1969), p. 100.

${ }^{12} \mathrm{~J}$. P. Gordon notes that power broadening occurs at still higher powers. This increases the hole width and the average force $\sim \sqrt{P}$.

${ }^{13}$ See Ref. 11, p. 166.
} 